\title{
Distribuição e raridade das espécies de Mezilaurus (Lauraceae) no Brasil
}

Distribution and rarity of Mezilaurus (Lauraceae) species in Brazil

\author{
Carlos H. Franciscon ${ }^{1,3}$ \& Izildinha S. Miranda ${ }^{2}$
}

\begin{abstract}
Resumo
Este estudo teve o objetivo de apresentar uma revisão sobre a distribuição conhecida das espécies de Mezilaurus ocorrentes no Brasil e investigar se a distribuição potencial e se o estado de conservação das espécies podem ser estudados com base nos dados existentes nos herbários. A distribuição potencial de Mezilaurus foi modelada através do sistema Biogeografia da Flora e Fungos do Brasil. Foi encontrado um total 804 registros de 16 espécies brasileiras de Mezilaurus. O percentual de perda total de registros foi muito grande $(58,3 \%)$, especialmente devido à falta de georreferenciamento e determinador $(56,4 \%)$. A análise da distribuição conhecida revelou novas informações para M. lindaviana, M. navalium, M. sprucei, M. subcordata, M. synandra e $M$. vanderwerffii. Os modelos de distribuição potencial apresentaram bons resultados para $M$. itauba, M. crassiramea e M. synandra; contudo foram fortemente influenciados pelo número de registros. A análise de raridade mostrou que cinquenta por cento das espécies apresentaram alto grau de raridade pois ocorrem em áreas e habitat restritos e populações escassas. A situação de conservação baseada na classificação de raridade usando os registros dos herbários apresentou falhas quanto à classificação do tamanho das populações, contudo, mostraram bons resultados sobre a amplitude geográfica e de habitat das espécies.
\end{abstract}

Palavras-chave: Amazônia, conservação, modelos de distribuição das espécies.

\begin{abstract}
This study aimed to present a review on known distribution of Mezilaurus species from Brazil and investigate whether the potential distribution and the conservation status of the species can be studied based on herbarium data. The potential distribution of Mezilaurus species was modeled using the Brazilian Floral and Fungal Biogeography system. A total of 804 records of 16 Brazilian species of Mezilaurus was found. The percentage of total loss of records was very high (58.3\%), especially due to lack of georeferencing and the sampling determiner (56.4\%). Analysis of the known distribution revealed new information for M. lindaviana, M. navalium, M. sprucei, $M$. subcordata, M. synandra and M. vanderwerffii. The potential distribution models showed good results for M. itauba, M. crassiramea and M. synandra; however, were strongly influenced by the number of records. The analysis of rarity showed that fifty percent of the species presented a high degree of rarity because they occur in restricted areas and habitat and scanty populations. The situation of conservation based on the classification of rarity using herbarium records showed some failures regarding the classification of the population size; however, they showed good results on the geographic range and habitat of the species.
\end{abstract}

Key words: Amazonian, conservation, species distribution modeling.

Veja material suplementar em <https://doi.org/10.6084/m9.figshare.6185336.v1>

\footnotetext{
${ }^{1}$ Instituto Nacional de Pesquisas da Amazônia, CBIO/CTIN, Av. André Araújo 2936, Petrópolis, C.P. 2223, 69080-971, Manaus, AM, Brasil.

${ }^{2}$ Universidade Federal Rural da Amazônia, Inst. Socioambiental e de Recursos Hídricos, Av. Tancredo Neves 2501, Montese, C.P. 917, 66077-530, Belém, PA, Brasil.

${ }^{3}$ Autor para correspondência: franciscon.carlos@inpa.gov.br
} 


\section{Introdução}

O gênero Mezilaurus Taub. (Lauraceae) é composto por 18 espécies de árvores ou arbustos, com distribuição restrita à região tropical da América do Sul, dos países amazônicos (Bolívia, Colômbia, Equador, Guiana, Guiana Francesa, Peru, Suriname, Venezuela) até o sudeste do Brasil (van der Werff 1987).

Dada a qualidade e durabilidade da madeira de Mezilaurus, as espécies são tradicionalmente usadas na construção de embarcações, movelaria e todos os tipos de construções duráveis (Lisboa et al. 1991); contudo, outros usos também têm sido destacados. Os óleos essenciais de algumas espécies [Mezilaurus duckei van der Werff e Mezilaurus itauba (Meisn.) Taub. ex Mez], além de aromáticos, têm demonstrado efeito positivo em ensaios biológicos no tratamento de bactérias, leveduras e protozoários (Izumi et al. 2009); M. itauba também tem propriedades antioxidantes (Yamaguchi et al. 2012). Uma substância isolada das folhas de Mezilaurus crassiramea (Meisn.) Taub. ex Mez ( $\gamma$-lactona di-hidroxilada) foi ativa no tratamento do câncer de próstata, rins e cólon (Martins 2014).

Como todas as Lauraceae, a complexidade morfológica e taxonômica são fatores que dificultam a identificação das espécies de Mezilaurus (van der Werff 1987; Quinet 2005, 2006; Alves 2011; Alves \& Souza 2012; BFG 2015). Associada à complexidade morfológica está a conversão de nomes populares aos nomes científicos (Baraloto et al. 2007; Procópio \& Secco 2008). Todas as espécies de Mezilaurus podem ser chamadas de itaúba (ita $=$ pedra; uba $=$ madeira $)$ e tinhorão (Alves \& Souza 2012; BFG 2015). Esses problemas dificultam o reconhecimento das populações e diminui a qualidade dos registros nos herbários (Gaston \& García-Viñas 2010; Hassemer et al. 2016), consequentemente dificultam análises mais precisas e acuradas sobre a distribuição das espécies.

Para obter mais registros de determinada espécie, escolher novas áreas prioritárias para conservação, entre outras ações que visam o planejamento e a conservação, podem ser utilizados os modelos de distribuição potencial das espécies como ferramenta de orientação (Miller \& Morgan 2011; Giannini et al. 2012; Addison et al. 2013). Contudo, esses modelos necessitam de dados em abundância e com precisão taxonômica para serem confiáveis (Cayuela et al. 2009).
Tendo em vista a importância econômica e o potencial de uso das espécies de Mezilaurus, este estudo tem o objetivo de apresentar uma revisão sobre a distribuição conhecida das espécies ocorrentes no Brasil e investigar se a distribuição potencial e se o estado de conservação das espécies podem ser estudados com base nos dados existentes nos herbários.

\section{Material e Métodos}

Inicialmente foram considerados o total de registros dos nomes válidos de Mezilaurus existentes no banco de dados da rede SpeciesLink ( $<$ http:// splink.cria.org.br $>$ ) e em outros herbários virtuais que possuem importantes coleções brasileiras (a lista dos herbários consultados está apresentada no Anexo 1 do Material Suplementar $<$ https://doi. org/10.6084/m9.figshare.6185336.v1>). Os nomes e sinônimos dos taxa de Mezilaurus do Brasil foram compilados a partir das revisões taxonômicas do gênero (van der Werff 1987; Alves 2011; Alves \& Souza 2012), BFG (2015) e The International Plant Names Index (<http://www.ipni.org $>)$.

Para a análise da distribuição potencial foram utilizados apenas os registros da rede SpeciesLink. Os registros (Rt) de cada espécie de Mezilaurus encontrados tiveram suas informações revisadas e foram filtrados (Filtro 1) apenas os registros com nomes válidos (Rv); com os registros válidos foi analisada a distribuição conhecida de Mezilaurus. Posteriormente, esses registros válidos foram novamente filtrados (Filtro 2), passando apenas aqueles com coordenadas geográfica distintas e com identificadores especialistas do grupo taxonômico (Rgi); com esses registros foi modelada a distribuição potencial das espécies, que considerou cada registro com coordenada distinta como um ponto de treino ou pixel (Px). Amostras provenientes de cultivos não foram consideradas. As coordenadas geográficas dos registros encontrados no SpeciesLink foram analisadas via aplicativo dataCleaning (DC) e quando possível e necessário georreferenciadas pelo aplicativo geoLoc, ambos aplicativos são livres e encontrados em <http:// splink.cria.org.br>.

A distribuição potencial de cada espécie foi modelada através do Sistema Biogeografia da Flora e Fungos do Brasil (Biogeo, <http://biogeo. inct.florabrasil.net/>), que utiliza o programa openModeller para produção dos modelos baseados em dados de presença da espécie, a partir das variáveis ambientais atribuídas a cada ponto de treino ou pixel. 
As variáveis ambientais consideradas foram oito: a elevação (variável topográfica) e sete variáveis bioclimáticas (Precipitação no trimestre mais úmido do ano; Precipitação no trimestre mais seco do ano; Precipitação no trimestre mais quente do ano; Precipitação no trimestre mais frio do ano; Variação média de temperatura ao longo do dia; Temperatura máxima no mês mais quente do ano; Temperatura mínima no mês mais frio do ano). Essas variáveis foram obtidas no banco de dados do Global Climate Data WorldClim $(<\mathrm{http}: / / \mathrm{www}$. worldclim.org $>$ ), na resolução espacial aproximada de $1 \mathrm{~km}^{2}$; e, são aquelas que se aplicam para a região tropical dentre as 19 variáveis apresentadas no WorldClim. As variáveis ambientais para cada espécie estão apresentadas no Anexo 2 do Material Suplementar <https://doi.org/10.6084/ m9.figshare.6185336.v1>.

Para analisar a distribuição potencial das espécies que possuíam maior ou igual a 10 pixels foi utilizado o modelo de consenso (Muñoz et al. 2011) baseado no valor médio de até cinco diferentes algoritmos (Maximum Entropy MaxEnt; Genetic Algorithm for Ruleset Prediction - Best Subsets - GARP BS; Distância Mahalanobis - DM; Ecological - Niche Factor Analysis - ENFA; Support Vector Machines - SVM) conforme recomendado por Marmion et al. (2009). O modelo de consenso apresenta níveis de concordância entre os algoritmos descritos em cores diferentes: vermelha (para cinco algoritmos), laranja (quatro), amarelo (três) e azul (menos que três). Se entre cinco e nove pixels, foi usado o Maximum Entropy (MaxEnt), de acordo com Phillips et al. (2006). Se menor que cinco pixels, foi gerado o modelo da Dissimilaridade Ambiental (DA), usando a Distância Euclidiana em relação ao ponto de ocorrência mais próximo, de acordo com Carpenter et al. (1993).

Para avaliar os modelos com 5 a 19 pixels de ocorrência foi utilizado a omissão interna e os mesmos foram testados com o procedimento de leave-one-out, seguido do teste de Poisson binomial unicaudal. Nos modelos com 20 ou mais pixels, além da omissão interna, também foram considerados a omissão externa média e a área sob a curva (Area Under the Curve - AUC).

A omissão é um tipo de erro que ocorre quando o modelo não prevê áreas que são ambientalmente adequadas para a espécie. A omissão interna foi calculada com os mesmos pontos utilizados na geração do modelo, e a omissão externa foi calculada com pontos que não foram utilizados na geração do modelo. A omissão externa não foi calculada para o modelo MaxEnt. A AUC (Area under the Curve) é a relação entre a área do modelo e a área de estudo para cada limiar da curva; seus valores foram calculados com validação cruzada do tipo 5-fold, recomendado para as espécies que apresentam entre 20 e 199 pixels (Phillips et al. 2006). Normalmente sugere-se que a omissão interna não ultrapasse $5 \%$, a omissão externa não ultrapasse 20\% (Peterson et al. 2008) e os valores de AUC sejam considerados muito bons (AUC $>$ $0,9)$, bons $(>0,8)$ e razoáveis $(>0,7)$ conforme recomendado por Swets (1988). Modelos com menos de 5 pixels de ocorrência não foram testados; eles servem apenas para indicar áreas, relativamente próximas e ambientalmente semelhantes, onde deveriam ser realizadas novas coletas botânicas.

$\mathrm{O}$ estado de conservação foi avaliado com base na classificação de raridade de Rabinowitz et al. (1986), que sugere oito categorias de raridade baseada nas informações simultâneas de alcance geográfico (largo ou estreito), especificidade de habitat (amplo ou restrito) e tamanho das populações locais (abundante ou escassa). Para categorizar a raridade das espécies de Mezilaurus foram modificados os critérios de Serrato et al. (2004): o alcance geográfico foi definido pelo número de pixels nos quais as espécies foram encontradas; espécies com estreito alcance geográfico foram aquelas localizadas em menos que 14 pixels, equivalente a no máximo $25 \%$ do número de pixels ocupados por M. itauba (56 pixels, maior quantidade de pixels entre as espécies de Mezilaurus). Para determinar a categoria de especificidade de habitat, foi considerado o número de tipos de vegetação nos quais as espécies foram encontradas; espécies com habitat restrito foram aquelas que ocorreram em apenas um tipo de vegetação. As informações sobre os habitat foram extraídas de van der Werff (1987), Alves (2011), BFG (2015) e dos rótulos das exsicatas dos herbários. A categoria do tamanho das populações locais foi definida pelo número de total de registros encontrado SpeciesLink (Tr); espécies com populações pequenas foram aquelas com menos que 72 registros, equivalente a no máximo $25 \%$ dos registros de $M$. itauba, (287, maior quantidade de registros dentre as espécies de Mezilaurus).

Os resultados encontrados na classificação de raridade das espécies foram comparados com aqueles apresentados na lista vermelha de espécies ameaçadas da IUCN (2015) e na lista vermelha da Flora do Brasil (RLBF 2016). 


\section{Resultados e Discussão}

Dados e perda de dados

São referidas para o Brasil 16 espécies de Mezilaurus, e para nove destas espécies foram encontrados 25 sinônimos válidos (Tab. 1). A relação de espécies da BFG (2015) apresenta 17 espécies do gênero, contudo uma delas não foi encontrada entre os registros analisados (Mezilaurus thoroflora van der Werrf).

Inicialmente foram encontrados 975 registros de Mezilaurus no Brasil, porém 171 destes foram pré-excluídos por serem identificados apenas ao nível de gênero. Assim, o número total de registros considerados foi de 804 ; destes, 36 foram excluídos por não apresentarem nomes válidos e outros 433 foram excluídos por não apresentarem georreferenciamento ou identificador especialista. Ao final, foram considerados 335 registros, agrupados em 145 pixels (Tab. 2).
Considerando as 16 espécies, o percentual de perda total de registros foi muito grande $(58,3 \%)$, especialmente devido à falta de georreferenciamento e determinador $(56,4 \%)$. Para Mezilaurus caatingae van der Werf e M. decurrens (Ducke) Kosterm. a perda foi total e em $M$. navalium (Allemão) Taub. ex Mez, M. synandra (Mez) Kosterm, M. subcordata (Ducke) Kosterm., M. lindaviana Schwacke \& Mez a perda foi superior a 70\%. Mezilaurus microphylla F.M.Alves \& V.C.Souza foi a única espécie que não apresentou perdas em nenhum dos filtros (Tab. 2).

A falta de georreferenciamento e de identificadores especializados é um problema recorrente nas exsicatas dos herbários. O georreferenciamento das amostras já é (ou deveria ser) item obrigatório para incorporação de exsicatas nos herbários; todavia a grande quantidade de material não identificado por especialistas, já destacada por outros autores (Ferreira \& Hopkins

Tabela 1 - Nomes e sinônimos das espécies brasileiras de Mezilaurus (Lauraceae).

Table 1 - Names and synonyms of Brazilian Mezilaurus (Lauraceae) species.

1. Mezilaurus caatingae van der Werf

2. Mezilaurus crassiramea (Meisn.) Taub. ex Mez

= Oreodaphne crassiramea Meisn.

= Mezia crassiramea (Meisn.) Kuntze

= Misanteca crassiramea (Meisn.) Benth.

= Silvia crassiramea (Meisn.) Mez

3. Mezilaurus decurrens (Ducke) Kosterm.

= Silvia decurrens Ducke

4. Mezilaurus duckei van der Werff

= Mezilaurus pyriflora van der Werff

5. Mezilaurus glabriantha F.M.Alves \& V.C.Souza

6. Mezilaurus itauba (Meisn.) Taub. ex Mez

= Acrodiclidium itauba Meisn.

= Acrodiclidium itauba var. amarella Meisn.

= Mezilaurus anacardioides (Spruce ex Meisn.)

Taub. ex Mez

= Oreodaphne hookeriana Meisn.

= Silvia polyantha $\mathrm{Mez}$

= Silvia rondonii Mez \& Hoehne

= Endiandra itauba (Meisn.) Benth.

= Mezia itauba (Meisn.) Kuntze

= Silvia itauba (Meisn.) Pax
7. Mezilaurus lindaviana Schwacke \& Mez

= Mezilaurus wurdackiana C.K. Allen

= Silvia duckei (A.Samp.) A.Samp.

= Misanteca duckei A.Samp.

8. Mezilaurus manausensis van der Werff

9. Mezilaurus micrantha van der Werff

10. Mezilaurus microphylla F.M.Alves \& V.C.Souza

11. Mezilaurus navalium (Allemão) Taub. ex Mez

= Silvia navalium Allemão

= Endiandra navalium (Allemão) Benth.

= Mezia navalium (Allemão) Kuntze

= Silvaea navalium (Allemão) Meisn

12. Mezilaurus sprucei (Meisn.) Taub. ex Mez =Acrodiclidium sprucei Meisn

13. Mezilaurus subcordata (Ducke) Kosterm. = Silvia subcordata Ducke

14. Mezilaurus synandra (Mez) Kosterm. = Silvia synandra $\mathrm{Mez}$

15. Mezilaurus revolutifolia F.M.Alves \& P.L.R.Moraes

16. Mezilaurus vanderwerffii F.M.Alves \& Baitello 
Tabela $2-\mathrm{Rt}=$ número total de registros; $\mathrm{Rv}=$ registros com nomes válidos; $\mathrm{Rgi}=$ registro georreferenciados e com determinador; $\mathrm{Px}=$ número de pixels; $\mathrm{F} 1$ = porcentagem de perda por falta de nomes válidos; $\mathrm{F} 2$ = porcentagem de perda por falta de coordenadas geográfica distintas e identificadores especialistas do grupo taxonômico; e porcentagem de perda total encontrados nos bancos de dados para confecção dos modelos de distribuição das espécies de Mezilaurus no Brasil. Table $2-\mathrm{Rt}=$ total number of records; $\mathrm{Rv}=$ records with valid names; $\mathrm{Rgi}=$ georeferenced species records and the name of the person who identified it; $\mathrm{Px}=$ number of pixels; F1 = percentage of loss in filter one; F2 = percentage of loss in filter two; and percentage of total loss found in databases used to build the models of Brazilian Mezilaurus species distribution.

\begin{tabular}{lccccccc}
\hline Espécies & $\mathbf{R t}$ & $\mathbf{R v}$ & $\mathbf{R g i}$ & $\mathbf{P x}$ & $\begin{array}{c}\text { Perda } \\
\text { (F1)(\%) }\end{array}$ & $\begin{array}{c}\text { Perda } \\
\text { (F2)(\%) }\end{array}$ & $\begin{array}{c}\text { Perda total } \\
\mathbf{( \% )}\end{array}$ \\
\hline Mezilaurus caatingae & 1 & 1 & 0 & 0 & 0,0 & 100,0 & 100,0 \\
Mezilaurus crassiramea & 104 & 102 & 46 & 26 & 1,9 & 54,9 & 55,8 \\
Mezilaurus decurrens & 13 & 13 & 0 & 0 & 0,0 & 100,0 & 100,0 \\
Mezilaurus duckei & 51 & 50 & 33 & 9 & 2,0 & 34,0 & 35,3 \\
Mezilaurus glabriantha & 11 & 8 & 4 & 2 & 27,3 & 50,0 & 63,6 \\
Mezilaurus itauba & 287 & 278 & 123 & 56 & 3,1 & 55,8 & 57,1 \\
Mezilaurus lindaviana & 77 & 70 & 21 & 6 & 9,1 & 70,0 & 72,7 \\
Mezilaurus manausensis & 24 & 24 & 16 & 3 & 0,0 & 33,3 & 33,3 \\
Mezilaurus micrantha & 16 & 16 & 13 & 6 & 0,0 & 18,8 & 18,8 \\
Mezilaurus microphylla & 1 & 1 & 1 & 1 & 0,0 & 0,0 & 0,0 \\
Mezilaurus navalium & 27 & 26 & 4 & 4 & 3,7 & 84,6 & 85,2 \\
Mezilaurus revolutifolia & 26 & 24 & 22 & 7 & 7,7 & 8,3 & 15,4 \\
Mezilaurus sprucei & 29 & 22 & 15 & 2 & 24,1 & 31,8 & 48,3 \\
Mezilaurus subcordata & 42 & 41 & 10 & 6 & 2,4 & 75,6 & 76,2 \\
Mezilaurus synandra & 72 & 70 & 18 & 10 & 2,8 & 74,3 & 75,0 \\
Mezilaurus vanderwerffii & 23 & 22 & 9 & 7 & 4,3 & 59,1 & 60,9 \\
\hline Total & 804 & 768 & 335 & 145 & 4,5 & 56,4 & 58,3 \\
\hline
\end{tabular}

2004; Hopkins 2007), é por um lado devido à grande quantidade de material oriundo de vários projetos de cunho ambiental, os quais não possuem em seu corpo técnico especialistas para a identificação do material e, por outro lado, devido ao pequeno corpo técnico especializado existente no mercado, fato inclusive que passa por políticas públicas de âmbito nacional.

Poucos registros nas coleções podem representar estreita distribuição geográfica das espécies; e ou porque possuem populações muito escassas; e ou porque são pouco coletadas, o que levaria a uma falsa raridade da espécie (Siqueira et al. 2009) e limitações na identificação de áreas de endemismo (Murray-Smith et al. 2009). Uma concentração das coletas botânicas restrita a poucas áreas (Hopkins 2007; Schulman et al. 2007) é às vezes confundida com áreas de alto endemismo, e isto é muito comum na Amazônia (Nelson et al. 1990).
Entretanto, mesmo em áreas com muitas coletas botânicas como a região de Manaus-AM, existe apenas um registro de Mezilaurus caatingae e poucos registros para $M$. manausensis van der Werff, M. micrantha van der Werff e M.sprucei (Meisn.) Taub. ex Mez, fato que sugere suas reais raridades; da mesma forma, M. glabriantha F.M.Alves \& V.C.Souza, que também é procedente de uma região bem coletada na Reserva da Vale do Rio Doce, em Minas Gerais e Espírito Santo. Ao contrário, M. microphylla só possui um registro, em área pouco coletada (TO), o que sugere a necessidade de um maior esforço de coleta. Já $M$. decurrens possui 13 registros válidos, mas nenhum deles foi georreferenciado ou identificado por especialista; essa espécie poderia ser priorizada pelos herbários para qualificar a identificação e o georrefenciamento. 


\section{Distribuição conhecida}

A distribuição geográfica conhecida das espécies de Mezilaurus no Brasil mostra um padrão predominante na Amazônia (Tab. 3). Apenas cinco espécies (M. glabriantha, M. microphylla, $M$. navalium, $M$. revolutifolia F.M.Alves \& P.L.R.Moraes e $M$. vanderwerffii) não possuem ocorrência conhecida na Amazônia.

A análise da distribuição conhecida revelou novas informações para seis espécies: (1) Mezilaurus lindaviana não tinha registro de ocorrência para Roraima (BFG 2015); porém, encontramos registros para este estado em Macajari, Estação Ecológica Ilha de Maracá, 12.VII.1986, D.G.Campbel 15658(MO), 19.IX.1987, W.Milliken 571 (MO), 7.X.1987, J.Pruski et al. 3402 (MO); (2) M. navalium não possui apenas uma ocorrência no Rio de Janeiro como citado por van der Werff (1987); em nosso estudo encontramos outro registro para Petrópolis, 2.II.1880, A.F.M.Glaziou, 12125 (IAC, MO); (3) a ocorrência de M. sprucei (Meisn.) Taub. ex Mez, no Acre, se baseia unicamente às

Tabela 3 - Distribuição geográfica das espécies de Mezilaurus do Brasil. As abreviações dos estados brasileiros são: $\mathrm{AC}=$ Acre; $\mathrm{AP}=$ Amapá; $\mathrm{AM}=$ Amazonas; $\mathrm{BA}=$ Bahia; $\mathrm{CE}=$ Ceará; $\mathrm{ES}=$ Espirito Santo; $\mathrm{GO}=\mathrm{Goiás} ; \mathrm{MA}$ = Maranhão; MT = Mato Grosso; MS = Mato Grosso do Sul; MG = Minas Gerais; PA = Pará; PI = Piauí; RJ = Rio de Janeiro; RS = Rio Grande do Sul; RN = Rio Grande no Norte; RO = Rondônia; RR = Roraima; TO = Tocantins. Table 3 - Geographical distribution of Brazilian Mezilaurus species. The abbreviations of the Brazilian states are: AC = Acre; $\mathrm{AP}=$ Amapá; $\mathrm{AM}=$ Amazonas; $\mathrm{BA}=$ Bahia $; \mathrm{CE}=$ Ceará; $\mathrm{ES}=$ Espirito Santo; $\mathrm{GO}=$ Goiás; $\mathrm{MA}=$ Maranhão; $\mathrm{MT}=$ Mato Grosso; $\mathrm{MS}=$ Mato Grosso do Sul; MG = Minas Gerais; PA = Pará; PI = Piauí; RJ = Rio de Janeiro; RS = Rio Grande do Sul; RN = Rio Grande no Norte; $\mathrm{RO}=$ Rondônia; $\mathrm{RR}=$ Roraima; $\mathrm{TO}=$ Tocantins.

\begin{tabular}{|c|c|c|c|c|c|}
\hline \multirow[t]{2}{*}{ Espécies } & \multicolumn{2}{|c|}{$\begin{array}{l}\text { Distribuição } \\
\text { conhecida }\end{array}$} & \multirow{2}{*}{$\begin{array}{l}\text { Distribuição } \\
\text { - Potencial }\end{array}$} & \multirow[t]{2}{*}{ Habitat } & \multirow{2}{*}{$\begin{array}{l}\text { Endereço do modelo } \\
\text { no Biogeo }\end{array}$} \\
\hline & Bioama & Estados & & & \\
\hline $\begin{array}{l}\text { Mezilaurus } \\
\text { caatingae }\end{array}$ & Amazônia & $\mathrm{AM}$ & $\begin{array}{l}\text { Dados insuficientes para } \\
\text { predição }\end{array}$ & $\begin{array}{l}\text { Campina sobre solos } \\
\text { arenosos da Amazônia }\end{array}$ & Não tem modelo \\
\hline $\begin{array}{l}\text { Mezilaurus } \\
\text { crassiramea }\end{array}$ & $\begin{array}{l}\text { Amazônia, } \\
\text { Cerrados }\end{array}$ & $\begin{array}{l}\text { AM, AP, } \\
\text { ES, GO, } \\
\text { MG, RO, } \\
\text { MS, MT, } \\
\text { TO }\end{array}$ & $\begin{array}{l}\text { O modelo consenso de } 5 \\
\text { algoritmos ampliou ás áreas } \\
\text { de ocorrência no entorno } \\
\text { dos pontos de coleta. Áreas } \\
\text { com condições ambientais } \\
\text { adequadas nos estados do } \\
\text { AC, MA, PA, RR }\end{array}$ & $\begin{array}{l}\text { Campo Rupestre, } \\
\text { Cerrado (lato sensu), } \\
\text { Floresta de Galeria, } \\
\text { Floresta Estacional } \\
\text { Semidecidual, Floresta } \\
\text { Ombrófila. Solo } \\
\text { arenoso e argiloso }\end{array}$ & $\begin{array}{l}<\text { http://biogeo.inct. } \\
\text { florabrasil.net/proc/15763>, } \\
\text { (miniatura do mapa Fig. 1a) }\end{array}$ \\
\hline $\begin{array}{l}\text { Mezilaurus } \\
\text { decurrens }\end{array}$ & Amazônia & $\mathrm{AM}$ & $\begin{array}{l}\text { Dados insuficientes para } \\
\text { predição }\end{array}$ & Floresta Ombrófila & Não tem modelo \\
\hline $\begin{array}{l}\text { Mezilaurus } \\
\text { duckei }\end{array}$ & Amazônia & $\mathrm{AM}$ & $\begin{array}{l}\text { O modelo MaxEnt restringiu } \\
\text { a ocorrência às áreas de } \\
\text { coletas no AM }\end{array}$ & $\begin{array}{l}\text { Floresta Ombrófila. } \\
\text { Solo argiloso, argilo- } \\
\text { arenoso e latosolo } \\
\text { amarelo }\end{array}$ & $\begin{array}{l}<\text { http://biogeo.inct. } \\
\text { florabrasil.net/proc/15763>, } \\
\text { (miniatura do mapa Fig. 2) }\end{array}$ \\
\hline $\begin{array}{l}\text { Mezilaurus } \\
\text { glabriantha }\end{array}$ & $\begin{array}{l}\text { Mata } \\
\text { Atlântica }\end{array}$ & ES & $\begin{array}{l}\text { O modelo de dissimilaridade } \\
\text { ambiental, ampliou a área de } \\
\text { ocorrência no ES }\end{array}$ & Floresta Ombrófila & $\begin{array}{l}<\text { http://biogeo.inct. } \\
\text { florabrasil.net/proc/15763>, } \\
\text { (miniatura do mapa Fig. 3) }\end{array}$ \\
\hline $\begin{array}{l}\text { Mezilaurus } \\
\text { itauba }\end{array}$ & Amazônia & $\begin{array}{l}\mathrm{AC}, \mathrm{AM}, \\
\mathrm{AP}, \mathrm{PA}, \\
\mathrm{RO}, \mathrm{RR}, \\
\mathrm{MT}\end{array}$ & $\begin{array}{l}\text { O modelo de consenso de } \\
5 \text { algoritmos ampliou as } \\
\text { áreas de ocorrência em } \\
\text { toda a Amazônia. Áreas } \\
\text { com condições ambientais } \\
\text { adequadas nos estados do } \\
\text { ES, MG e MS }\end{array}$ & $\begin{array}{l}\text { Floresta Ombrófila, } \\
\text { Floresta de Várzea } \\
\text { e Igapó. Campina } \\
\text { aberta, Campinarana } \\
\text { arbórea, Solo argiloso, } \\
\text { arenoso e latossolo } \\
\text { amarelo franco }\end{array}$ & $\begin{array}{l}<\text { http://biogeo.inct. } \\
\text { florabrasil.net/proc/15767>, } \\
\text { (miniatura do mapa Fig. 1) }\end{array}$ \\
\hline
\end{tabular}




\begin{tabular}{|c|c|c|c|c|c|}
\hline \multirow{2}{*}{ Espécies } & \multicolumn{2}{|c|}{$\begin{array}{l}\text { Distribuição } \\
\text { conhecida }\end{array}$} & \multirow{2}{*}{$\begin{array}{l}\text { Distribuição } \\
\text { Potencial }\end{array}$} & \multirow[t]{2}{*}{ Habitat } & \multirow{2}{*}{$\begin{array}{l}\text { Endereço do modelo } \\
\text { no Biogeo }\end{array}$} \\
\hline & Bioama & Estados & & & \\
\hline $\begin{array}{l}\text { Mezilaurus } \\
\text { lindaviana }\end{array}$ & $\begin{array}{l}\text { Amazônia, } \\
\text { Cerrado }\end{array}$ & $\begin{array}{l}\mathrm{AM}, \mathrm{AP} \\
\mathrm{PA}, \mathrm{RR}\end{array}$ & $\begin{array}{l}\text { O modelo MaxEnt ampliou } \\
\text { as áreas de ocorrência no } \\
\text { AM, AP, PA e RR }\end{array}$ & $\begin{array}{l}\text { Cerrado (lato sensu), } \\
\text { Floresta Ombróflia }\end{array}$ & $\begin{array}{l}<\text { http://biogeo.inct. } \\
\text { florabrasil.net/proc/15768>, } \\
\text { (miniatura do mapa Fig. 2) }\end{array}$ \\
\hline $\begin{array}{l}\text { Mezilaurus } \\
\text { manausensis }\end{array}$ & Amazônia & $\mathrm{AM}$ & $\begin{array}{l}\text { O modelo de dissimilaridade } \\
\text { ambiental, ampliou as áreas } \\
\text { de ocorrência para o AM }\end{array}$ & $\begin{array}{l}\text { Floresta Ombrófila. } \\
\text { Latossolo amarelo }\end{array}$ & $\begin{array}{l}<\text { http://biogeo.inct. } \\
\text { florabrasil.net/proc/15769>, } \\
\text { (miniatura do mapa Fig. 3) }\end{array}$ \\
\hline $\begin{array}{l}\text { Mezilaurus } \\
\text { micrantha }\end{array}$ & Amazônia & $\mathrm{AM}$ & $\begin{array}{l}\text { O modelo MaxEnt restringiu } \\
\text { a ocorrência aos pontos de } \\
\text { coletas no AM }\end{array}$ & $\begin{array}{l}\text { Floresta Ombrófila. } \\
\text { Latossolo amarelo, } \\
\text { argiloso }\end{array}$ & $\begin{array}{l}<\text { http://biogeo.inct. } \\
\text { florabrasil.net/proc/15770>, } \\
\text { (miniatura do mapa Fig. 2) }\end{array}$ \\
\hline $\begin{array}{l}\text { Mezilaurus } \\
\text { microphylla }\end{array}$ & Cerrado & TO & $\begin{array}{l}\text { O modelo de dissimilaridade } \\
\text { ambiental, indicou como } \\
\text { mais adequadas as áreas no } \\
\text { entorno da coleta em TO }\end{array}$ & $\begin{array}{l}\text { Cerrado lato sensu, } \\
\text { Floresta Estacional } \\
\text { Semidecidual }\end{array}$ & $\begin{array}{l}<\text { http://biogeo.inct. } \\
\text { florabrasil.net/proc/15771>, } \\
\text { (miniatura do mapa Fig. 3) }\end{array}$ \\
\hline $\begin{array}{l}\text { Mezilaurus } \\
\text { navalium }\end{array}$ & $\begin{array}{l}\text { Mata } \\
\text { Atlântica }\end{array}$ & RJ, MS & $\begin{array}{l}\text { O modelo de dissimilaridade } \\
\text { ambiental, indicou como } \\
\text { mais adequadas as áreas } \\
\text { no entorno dos pontos de } \\
\text { coleta. O norte de MT é uma } \\
\text { área adequada para ampliar } \\
\text { esforços de coletas }\end{array}$ & Floresta Ombrófila & $\begin{array}{l}<\text { http://biogeo.inct. } \\
\text { florabrasil.net/proc/15772>, } \\
\text { (miniatura do mapa Fig. 3) }\end{array}$ \\
\hline $\begin{array}{l}\text { Mezilaurus } \\
\text { revolutifolia }\end{array}$ & $\begin{array}{l}\text { Mata } \\
\text { Atlântica }\end{array}$ & BA & $\begin{array}{l}\text { O modelo MaxEnt indicou } \\
\text { grande parte do Brasil como } \\
\text { área adequada; contudo } \\
\text { o modelo mostrou alta } \\
\text { omissão interna, por isso } \\
\text { não foi considerado como } \\
\text { adequado }\end{array}$ & $\begin{array}{l}\text { Floresta Estacional } \\
\text { Semidecidual, Floresta } \\
\text { Ombrófila, Floresta de } \\
\text { restinga }\end{array}$ & $\begin{array}{l}<\text { http://biogeo.inct. } \\
\text { florabrasil.net/proc/15773>, } \\
\text { (miniatura do mapa Fig. 2) }\end{array}$ \\
\hline $\begin{array}{l}\text { Mezilaurus } \\
\text { sprucei }\end{array}$ & Amazônia & $\mathrm{AM}$ & $\begin{array}{l}\text { O modelo de dissimilaridade } \\
\text { ambiental restringiu aos } \\
\text { pontos de coletas no AM }\end{array}$ & $\begin{array}{l}\text { Floresta de Terra } \\
\text { Firme, Floresta de } \\
\text { Várzea e de Igapó }\end{array}$ & $\begin{array}{l}<\text { http://biogeo.inct. } \\
\text { florabrasil.net/proc/15774>, } \\
\text { (miniatura do mapa Fig. 3) }\end{array}$ \\
\hline $\begin{array}{l}\text { Mezilaurus } \\
\text { subcordata }\end{array}$ & Amazônia & $\begin{array}{l}\mathrm{AC}, \mathrm{AM}, \\
\mathrm{PA}, \mathrm{RO}, \\
\mathrm{RR}, \mathrm{MT}\end{array}$ & $\begin{array}{l}\text { O modelo MaxEnt } \\
\text { confirmou a distribuição } \\
\text { geográfica conhecida }\end{array}$ & $\begin{array}{l}\text { Floresta Ombrófila. } \\
\text { Latossolo amarelo } \\
\text { franco argiloso, } \\
\text { arenoso, argilo- } \\
\text { arenoso }\end{array}$ & $\begin{array}{l}<\text { http://biogeo.inct. } \\
\text { florabrasil.net/proc/15775>, } \\
\text { (miniatura do mapa Fig. } 2 \text { ) }\end{array}$ \\
\hline $\begin{array}{l}\text { Mezilaurus } \\
\text { synandra }\end{array}$ & $\begin{array}{l}\text { Amazônia, } \\
\text { Cerrado, } \\
\text { Mata } \\
\text { Atlântica }\end{array}$ & $\begin{array}{l}\text { AM, BA, } \\
\text { PA }\end{array}$ & $\begin{array}{l}\text { O modelo consenso de } \\
2 \text { algoritmos (Garp e } \\
\text { MaxEnt) ampliou as áreas } \\
\text { de ocorrência próximas aos } \\
\text { locais de coletas, com alto } \\
\text { potencial para outros estados } \\
\text { da Amazônia (AC, AP, PA, } \\
\text { RO e RR) }\end{array}$ & $\begin{array}{l}\text { Área antrópica, } \\
\text { Campinarana, Floresta } \\
\text { de Terra Firme, } \\
\text { Floresta de Várzea, } \\
\text { Floresta Ciliar, } \\
\text { Capoeira Aberta, } \\
\text { Vegetação Secundária. } \\
\text { Solo arenoso }\end{array}$ & $\begin{array}{l}<\text { http://biogeo.inct. } \\
\text { florabrasil.net/proc/15776>, } \\
\text { (miniatura do mapa Fig. 2) }\end{array}$ \\
\hline $\begin{array}{l}\text { Mezilaurus } \\
\text { vanderwerffii }\end{array}$ & $\begin{array}{l}\text { Cerrado e } \\
\text { Pantanal }\end{array}$ & MT, MS & $\begin{array}{l}\text { O modelo MaxEnt } \\
\text { confirmou a distribuição } \\
\text { geográfica conhecida }\end{array}$ & $\begin{array}{l}\text { Floresta Estacional } \\
\text { Semidecidual, Cerrado } \\
\text { (lato sensu). Relevo } \\
\text { plano, solo arenoso, } \\
\text { argilo-arenoso }\end{array}$ & $\begin{array}{l}<\text { http://biogeo.inct. } \\
\text { florabrasil.net/proc/1577>, } \\
\text { (miniatura do mapa Fig. 2) }\end{array}$ \\
\hline
\end{tabular}


coletas de D.G. Campbel et al. 6867, 6792, 7037, 7753, 7825 (MO), todas elas realizadas em Santa Luzia, Reserva do INCRA, que além de serem identificadas como Mezilaurus c.f. sprucei (Meisn.) Taub. ex Mez., não têm o nome do identificador; portanto, a ocorrência neste estado foi tida como incerta; (4) M. subcordata, embora sua presença em Roraima seja citada pela BFG (2015), baseia-se apenas em 14.II.20014, J.G.Soler \& E.F.Barbosa 214 (ESA), sem identificador, portanto a ocorrência neste estado também foi tida como incerta; (5) M. synandra, é citada pela BFG (2015), como ocorrente no Amazonas, Bahia, Goiás e Tocantins; contudo não foram encontrados registros para Goiás ou Tocantins; por outro lado, encontramos um registro indicando ocorrência da espécie no Pará, 30.I.2013, I.L.Amaral 3631 (INPA); (6) $M$. vanderwerffii F.M.Alves \& Baitello possui um único registro encontrado em Mato Grosso em 1978, C.Costa 180796 (MO), no entanto este registro não possui identificador, logo a ocorrência neste estado também foi tida como incerta.

\section{Distribuição potencial}

Os modelos consensos, aplicados nas espécies com mais de 20 pixels (Mezilaurus itauba e $M$. crassiramea), refletiram bem os resultados dos algoritmos utilizados e apresentaram valores de omissão interna abaixo de $5 \%$, omissão externa média abaixo de $20 \%$ e AUC maior que 0,9. Da mesma forma, o modelo consenso para $M$. synandra (com 10 pixels) apresentou baixa omissão interna e probabilidade menor que 0,01 (Tab. 4). Esses resultados estão ligados ao elevado número de pixels, que por sua vez depende do número de registros; o que eleva a acurácia e números de acertos dos modelos (Siqueira et al. 2009).

A distribuição potencial de Mezilaurus itauba ampliou a ocorrência conhecida (AC, AM, AP, MT, PA, RO e RR) para toda a Amazônia (Fig. 1a; Tab. 3); sem ocorrência conhecida, mas com condições ambientais adequadas existem áreas nos estados do ES, MG e MS; essas áreas poderiam ser passíveis de plantios, uma vez que M. itauba é uma espécie de alto valor comercial.

Mezilaurus crassiramea teve a distribuição conhecida (AM, AP, ES, GO, MG, RO, MS, MT e TO) ampliada no entorno dos pontos de coleta, na Amazônia e Cerrados (TO), podendo ocorrer também no AC, MA, PA e RR, pois nesses estados existem áreas com condições ambientais favoráveis a essa espécie (Fig. 1b; Tab. 3).

Mezilaurus synandra também apresentou ampliação da área de ocorrência nos locais de coletas já conhecidos (AM, BA, PA), e apontou um potencial de ocorrência em outros estados da Amazônia (AC, AP, PA, RO e RR), além de algumas áreas com condições ambientais adequadas no MS, RS e na faixa litorânea do ES até o RN (Fig. 1c); contudo, essas áreas podem ser distorções geradas pela escala de estudo, pois não há indicação de ocorrência dessa espécie nessas áreas. Como o modelo de $M$. synandra foi elaborado com apenas 10 pixels, um estudo mais detalhado, em uma escala menor é recomendado para que se confirme sua distribuição potencial. A modelagem estatística não é eficaz quando poucos pontos de dados estão disponíveis, pois os modelos não conseguem produzir previsões confiáveis (Cayuela et al. 2009).

Os modelos MaxEnt aplicado às seis espécies com número de pixels entre 5 e 9, apresentou bons resultados somente para Mezilaurus lindaviana, pois apresentou zero de omissão interna e probabilidade menor que 0,01. Para esta espécie, a distribuição conhecida (AM, AP, PA, RR) também foi ampliada pela modelagem, com potencial para exploração no entorno dos pontos de coleta (Fig. 1d; Tab. 3); assim como para $M$. synandra, a distribuição potencial precisa ser estudada em uma escala mais detalhada para garantir maior qualidade dos modelos, uma vez que essa espécie apresentou poucos pixels na escala desse estudo.

Nas demais espécies com número de pixels entre 5 e 9 a omissão interna foi maior que 5\% ou a probabilidade maior que 0,01 (Tab. 4); por isso, os modelos para Mezilaurus duckei, M. revolutifolia, $M$. vanderwerffii, $M$. micrantha e M. subcordata, assim como os modelos para as espécies que apresentam menos que 5 pixels ( $M$. navalium, M. manausensis, $M$. glabriantha, $M$. sprucei e $M$. microphylla) devem ser realizados em uma escala mais acurada, de forma a se obter mais pixels, garantindo assim maior qualidade nos modelos (Tab. 3).

Contudo, vale destacar que algumas espécies possuem poucos pixels devido ao baixo número de registros, e para elas, os modelos devem ser usados como ferramentas para identificar áreas com condições ambientais favoráveis às espécies; onde novas coletas possam ser realizadas e suas populações 
Tabela 4 - Indicadores de qualidade dos modelos aplicados nas espécies de Mezilaurus do Brasil. A porcentagem da omissão interna (Oi) comum a todos os modelos. Para cada modelo é apresentado a área sob a curva (AUC) e a omissão externa média (Om). Para as espécies com menos de 20 pixels é apresentado a Oi e o o valor da probabilidade oriundo do teste de Poisson-binomial unicaudal, para o modelo MaxEnt; e para as espécies com 1 a 4 pixels é apresentado apenas o valor da Distância Euclideana Máxima (DEM) encontrado para o Modelo de Dissimilaridade Ambiental. Table 4 - Quality indicators of the models applied to the Brazilian Mezilaurus species. The Intrinsic omission rate (Oi) is common to all models. For each model the area under the curve (AUC) and the average external omission (Om) are presented. For species with less than 20 pixels, the Oi and the probability value from the Poisson-binomial unicaudal test are presented for the MaxEnt model; And for species with 1 to 4 pixels only the maximum Euclidean Distance (DEM) value found for the Environmental Dissimilarity Model is presented.

\begin{tabular}{|c|c|c|c|c|c|c|c|c|c|c|c|c|c|}
\hline \multirow{3}{*}{ Espécies } & \multicolumn{13}{|c|}{ Modelo/Algoritmo } \\
\hline & \multirow{2}{*}{$\begin{array}{c}\text { Oi } \\
(\%)\end{array}$} & \multicolumn{2}{|c|}{ Consenso } & \multicolumn{2}{|c|}{ SVM } & \multicolumn{2}{|c|}{ ENFA } & \multicolumn{2}{|c|}{ GARP BS } & \multicolumn{2}{|c|}{ MaxEnt } & \multicolumn{2}{|c|}{ Mahalanobis } \\
\hline & & AUC & $\mathrm{Om}$ & AUC & $\mathrm{Om}$ & AUC & $\mathrm{Om}$ & AUC & $\mathrm{Om}$ & AUC & $\mathrm{Om}$ & AUC & $\mathrm{Om}$ \\
\hline & \multicolumn{13}{|c|}{ Espécies com 20 ou mais pixels } \\
\hline Mezilaurus itauba & 0 & 0,97 & 3,6 & 0,84 & 8,8 & 0,93 & 3,3 & 0,95 & 7,3 & 0,95 & 1,8 & 0,9 & 17,9 \\
\hline \multirow[t]{2}{*}{ Mezilaurus crassiramea } & 3,8 & 0,92 & 15,3 & 0,78 & 39,3 & 0,91 & 4 & 0,94 & 12 & 0,98 & 11,3 & 0,82 & 34,7 \\
\hline & \multicolumn{13}{|c|}{ Espécie com 10 a 19 pixels } \\
\hline \multirow[t]{2}{*}{ Mezilaurus synandra } & 0 & \multicolumn{2}{|c|}{$<0,01$} & - & - & - & - & \multicolumn{2}{|c|}{$<0,01$} & \multicolumn{2}{|c|}{$<0,01$} & - & - \\
\hline & \multicolumn{13}{|c|}{ Espécies com 5 a 9 pixels } \\
\hline Mezilaurus duckei & 11,1 & - & - & - & - & - & - & & - & $<0$ & 01 & - & - \\
\hline Mezilaurus revolutifolia & 14,3 & - & - & - & - & - & - & & - & $<0$ & 01 & - & - \\
\hline Mezilaurus vanderwerffii & 14,3 & - & - & - & - & - & - & & - & $=0$, & 08 & - & - \\
\hline Mezilaurus lindaviana & 0 & - & - & - & - & - & - & & - & $<0$, & & - & - \\
\hline Mezilaurus micrantha & 16,7 & - & - & - & - & - & - & & - & $<0$ & & - & - \\
\hline \multirow[t]{2}{*}{ Mezilaurus subcordata } & 0 & - & - & - & - & - & - & & - & $=0$, & & - & - \\
\hline & \multicolumn{13}{|c|}{ Espécies com 1 a 4 pixels } \\
\hline Mezilaurus navalium & \multicolumn{13}{|c|}{ Modelo de Dissimilaridade Ambiental: $\mathrm{DEM}=0.1$} \\
\hline Mezilaurus manausensis & \multicolumn{13}{|c|}{ Modelo de Dissimilaridade Ambiental: $\mathrm{DEM}=0.1$} \\
\hline Mezilaurus glabriantha & \multicolumn{13}{|c|}{ Modelo de Dissimilaridade Ambiental: $\mathrm{DEM}=0.1$} \\
\hline Mezilaurus sprucei & \multicolumn{13}{|c|}{ Modelo de Dissimilaridade Ambiental: DEM = 0.1} \\
\hline \multirow[t]{2}{*}{ Mezilaurus microphylla } & \multicolumn{13}{|c|}{ Modelo de Dissimilaridade Ambiental: DEM = 0.1} \\
\hline & \multicolumn{13}{|c|}{ Espécies com zero pixel } \\
\hline Mezilaurus caatingae & \multicolumn{13}{|c|}{ Sem modelo } \\
\hline Mezilaurus decurrens & \multicolumn{13}{|c|}{ Sem modelo } \\
\hline
\end{tabular}

possam ser avaliadas, conforme recomendado por Pearson et al. (2007). Na verdade, para todas as espécies aqui estudadas, a previsão de ocupação potencial das espécies é considerada mais importante do que a precisão do modelo (Rupprecht et al. 2011), uma vez que os modelos foram executados somente com dados de presença (Pearson 2007).

Os mapas de distribuição potencial de todas as espécies estão públicos no Biogeo e os seus endereços eletrônicos foram apresentados na Tabela 3.

\section{Raridade e conservação}

A análise de raridade mostrou que apenas duas espécies (Mezilaurus crassiramea, M. itauba) não apresentam nenhum nível de raridade (Tab. 5); contudo essas espécies já estão na lista vermelha da IUCN (2015), especialmente pela redução de suas populações locais. M. itauba é uma espécie de alto valor comercial devido à sua madeira, e foi muito explorada em toda a Amazônia (Amaral et al. 2012; Garcia et al. 2012). Para essas espécies a metodologia utilizada neste estudo para medir o tamanho das populações através do número 


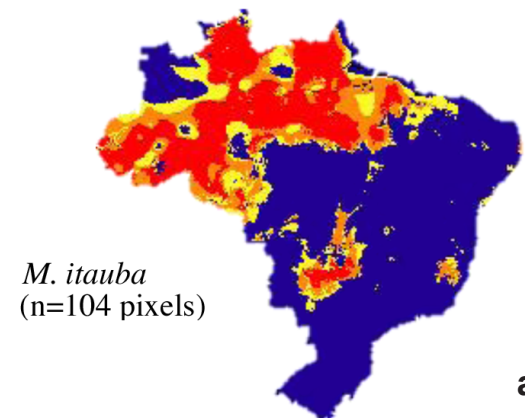

a

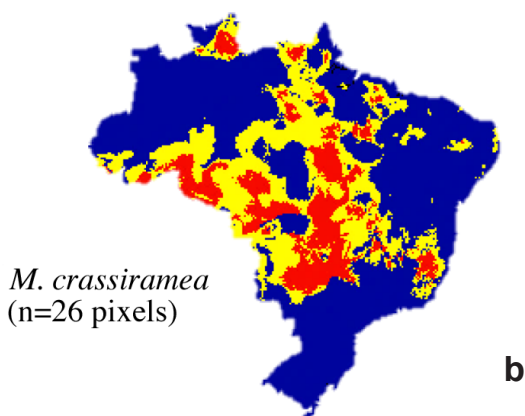

b

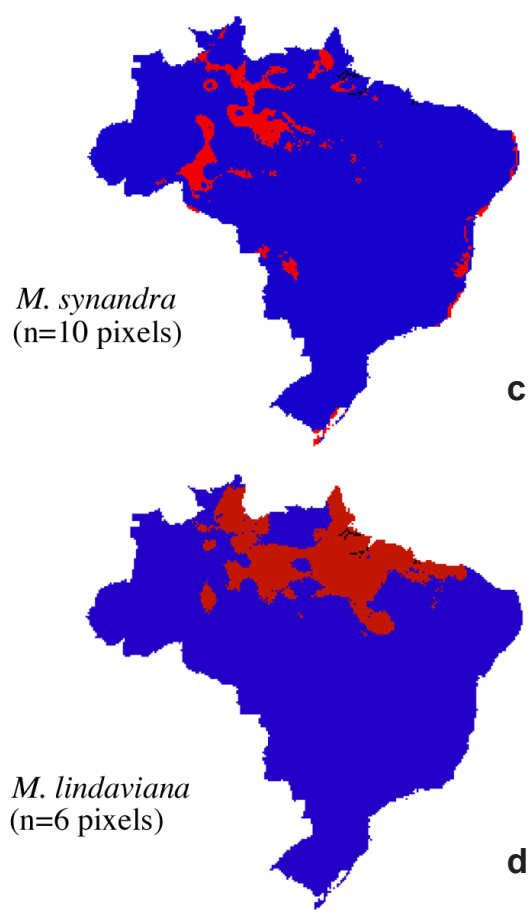

Figura 1 - a-d. Distribuição potencial das espécies de Mezilaurus - a,b. as cores representam áreas de concordância entre os algoritmos: vermelho = 5 algoritmos; laranja $=4$ algoritmos; amarelo $=3$ algoritmos; c,d. azul = menos de 3 algoritmos.

Figure 1 -a-d. Potential distribution of species of Mezilaurus -a,b. the colors represent areas of agreement between algorithms: red = 5 algorithms; orange $=4$ algorithms; yellow $=3$ algorithms; c,d . blue $=$ less than 3 algorithms. de registros não correspondeu à realidade. Nem sempre há uma correlação linear entre abundância das espécies e número de registros nos herbários; contudo, Serrato et al. (2004) não teve dificuldades em usar essa metodologia para analisar a distribuição do genus Ficus (Moraceae) no México.

Cinquenta por cento das espécies (Mezilaurus caatingae, M. decurrens, M. duckei, M. glabriantha, M. manausensis, $M$. micrantha, $M$. navalium, $M$. subcordata e $M$. vanderwerffii) apresentaram alto grau de raridade, pois ocorrem em áreas e habitat restritos e populações escassas. Dentre estas espécies, M. navalium já está presente nas listas da RLFB (2015) e IUCN (2015), o que confirma nossos resultados (Tab. 5); contudo, as demais espécies não foram avaliadas pelas listas vermelhas. Mesmo desconsiderando os resultados do tamanho das populações, a restrição geográfica e de habitat apresentado por essas espécies revela a necessidade de uma análise mais detalhada delas pelas listas vermelhas. Em especial destacamos M. caatingae, pois há mais de 60 anos não houve qualquer registro da espécie, o que confirma seu alto grau de raridade.

\section{Conclusão}

A análise da distribuição conhecida revelou novas áreas de ocorrência para Mezilaurus lindaviana, M. navalium e M. synandra.

A maioria dos registros (58\%) encontrados nos herbários é imprecisa, ou por falta de nomes válidos ou por falta de georreferenciamento e identificador especializado. Devido a esses problemas, apenas 4 das 16 espécies analisadas apresentaram bons resultados nos modelos de distribuição espacial (Mezilaurus itauba, M. crassiramea, M. synandra e M. lindaviana).

A situação de conservação baseada na classificação de raridade usando os registros dos herbários apresentou falhas quanto $a ̀$ classificação do tamanho das populações, contudo mostraram bons resultados sobre a amplitude geográfica e de habitat das espécies.

\section{Agradecimentos}

Ao Centro de Referência em Informação Ambiental-CRIA e ao Biogeografia da Flora e Fungos do Brasil-BIOGEO, especialmente a Renato Di Giovanni, as valiosas contribuições para realização deste estudo. Este estudo foi financiado pela Fundação de Amparo à Pesquisa do Estado do Amazonas (FAPEAM). 
Tabela 5 - Categorias de raridade e a situação de conservação das espécies de Mezilaurus do Brasil (EN = ameaçada; $\mathrm{NE}=$ não avaliada; $\mathrm{NT}=$ em risco de extinção; $\mathrm{CR}=$ criticamente ameaçada; $\mathrm{VU}=$ vulnerável).

Table 5 - Categories of rarity and conservation status of Brazilian Mezilaurus species $(\mathrm{EN}=$ endangered; $\mathrm{NE}=$ not evaluated; $\mathrm{NT}=$ near endangered; $\mathrm{CR}=$ critically endangered; $\mathrm{VU}=$ vulnerable).

\begin{tabular}{lccllll}
\hline \multirow{2}{*}{ Populações } & \multirow{2}{*}{$\begin{array}{l}\text { RLBF } \\
\text { (2016) }\end{array}$} & \multirow{2}{*}{$\begin{array}{l}\text { IUCN } \\
\text { (2015) }\end{array}$} & Distribuição & Habitat & Populações & Classificação \\
\hline Mezilaurus caatingae & $\mathrm{NE}$ & $\mathrm{NE}$ & Estreita & Restrito & Escassa & Maior nível de raridade \\
Mezilaurus crassiramea & $\mathrm{NT}$ & $\mathrm{CR}$ & Larga & Amplo & Abundante & Sem raridade \\
Mezilaurus decurrens & $\mathrm{NE}$ & $\mathrm{NE}$ & Estreita & Restrito & Escassa & Maior nível de raridade \\
Mezilaurus duckei & $\mathrm{NE}$ & $\mathrm{NE}$ & Estreita & Restrito & Escassa & Maior nível de raridade \\
Mezilaurus glabriantha & $\mathrm{NE}$ & $\mathrm{NE}$ & Estreita & Restrito & Escassa & Maior nível de raridade \\
Mezilaurus itauba & $\mathrm{VU}$ & $\mathrm{VU}$ & Larga & Amplo & Abundante & Sem raridade \\
Mezilaurus lindaviana & $\mathrm{NE}$ & $\mathrm{NE}$ & Estreita & Amplo & Escassa & Raridade intermediária \\
Mezilaurus manausensis & $\mathrm{NE}$ & $\mathrm{NE}$ & Estreita & Restrito & Escassa & Maior nível de raridade \\
Mezilaurus micrantha & $\mathrm{NE}$ & $\mathrm{NE}$ & Estreita & Restrito & Escassa & Maior nível de raridade \\
Mezilaurus microphylla & $\mathrm{NE}$ & $\mathrm{NE}$ & Estreita & Amplo & Escassa & Raridade preocupante \\
Mezilaurus navalium & $\mathrm{EN}$ & $\mathrm{VU}$ & Estreita & Restrito & Escassa & Maior nível de raridade \\
Mezilaurus revolutifolia & $\mathrm{NE}$ & $\mathrm{NE}$ & Estreita & Amplo & Escassa & Raridade intermediária \\
Mezilaurus sprucei & $\mathrm{NE}$ & $\mathrm{NE}$ & Estreita & Amplo & Escassa & Raridade intermediária \\
Mezilaurus subcordata & $\mathrm{NE}$ & $\mathrm{NE}$ & Estreita & Restrito & Escassa & Maior nível de raridade \\
Mezilaurus synandra & $\mathrm{NE}$ & $\mathrm{NE}$ & Estreita & Amplo & Abundante & Menor nível de raridade \\
Mezilaurus vanderwerffii & $\mathrm{NE}$ & $\mathrm{NE}$ & Estreita & Restrito & Escassa & Maior nível de raridade \\
\hline
\end{tabular}

\section{Referências}

Addison PFE, Rumpff L, Bau SS, Carey JM, Chee YE, Jarrad FC, McBride MF \& Burgman MA (2013) Practical solutions for making models indispensable in conservation decision-making. Diversity and Distributions 19: 490-502.

Alves FM \& Souza VC (2012) Two new species of Mezilaurus (Lauraceae) from Brazil. Brittonia 64: 257-262.

Alves FM (2011) Estudo taxonômico e filogenético de Mezilaurus Taub. (Lauraceae) lato sensu e restabelecimento de Clinostemon Kuhlm. \& A. Samp. Tese de Doutorado. Universidade de São Paulo, São Paulo. 234p.

Amaral DD, Vieira ICG, Salomão RP, Almeida SS \& Jardim MAG (2012) The status of conservation of urban forests in eastern Amazonia. Brazilian Journal of Biology 72: 257-265.

Baraloto C, Ferreira E, Rockwell C \& Walthier F (2007) Limitations and applications of parataxonomy for community forest management in southwestern Amazonia. Ethnobotany Research and Applications 5: 77-84.

BFG - The Brazil Flora Group (2015) Growing knowledge: an overview of seed plant diversity in Brazil. Rodriguésia 66: 1085-1113.

Carpenter G, Gillison AN \& Winter J (1993) DOMAIN: A flexible modeling procedure for mapping potential distributions of animals and plants. Biodiversity and Conservation 2: 667-680.

Cayuela L, Golicher DJ, Newton AC, Kolb M, Alburquerque FS, Arets EJMM, Alkemade JRM \& Pérez AM (2009) Species distribution modeling in the tropics: problems, potentialities, and the role of biological data for effective species conservation. Tropical Conservation Science 2: 319-352.

Ferreira GC \& Hopkins MJG (2004) Manual de identificação botânica e anatômica-angelim. Embrapa Amazônia Ocidental, Belém. 112p. 
Garcia FM, Manfio DR, Sansígolo CA \& Magalhães PAD (2012) Rendimento no desdobro de toras de itaúba (Mezilaurus itauba) e tauari (Couratari guianensis) segundo a Classificação da Qualidade da Tora. Floresta e Ambiente 19: 468-474.

Gastón A \& García-Viñas JI (2010) Updating coarsescale species distribution models using small fine-scale samples. Ecological Modelling 221: 2576-2581.

Giannini TC, Siqueira MF, Acosta AL, Barreto FCC, Saraiva AM \& Alves-dos-Santos I (2012) Desafios atuais da modelagem preditiva de distribuição de espécies. Rodriguésia 63: 733749.

Hassemer G, Giovanni R \& Trevisan R (2016) The use of potential distribution models in the study of the distribution and conservation status of plants: The case of Plantago L. (Plantaginaceae) in Brazil. Journal of the Torrey Botanical Society 143: 38-49.

Hopkins MJ (2007) Modelling the known and unknown plant biodiversity of the Amazon Basin. Journal of Biogeography 34: 1400-1411.

IUCN (2015) The IUCN red list of threatened species. Version 2015-4. Disponível em <http://www. iucnredlist.org > . Acesso em 15 março 2016.

Izumi E, Valdez RH, Alcântara JM, Yamaguchi KKL, Ueda-Nakamura T, Dias Filho BP, Veiga-Junior VF \& Nakamura CV (2009) Antiprotozoal, antibacterial and antifungal activities of plants of the Lauraceae family collected in the Brazilian Amazon rainforest. Planta Médica 75: PD9.

Lisboa PLB, Terezo EFM \& Silva JS (1991) Madeiras amazônicas: considerações sobre exploração, extinção e conservação. Boletim do Museu Paraense Emílio Goeldi. Série Botânica 7: 521-542.

Marmion M, Parviainen M, Luoto M, Heikkinen RK \& Thuiller W (2009) Evaluation of consensus methods in predictive species distribution modelling. Diversity and Distributions 15: 59-69.

Martins LAV (2014) Avaliação do potencial anticâncer de espécies vegetais de Mato Grosso do Sul. Tese de Doutorado. Universidade Federal do Mato Grosso do Sul, Campo Grande. 82p.

Miller JS \& Morgan HAP (2011) Assessing the effectiveness of Madagascar's changing protected areas system: a case study of threatened Boraginales. Oryx 45: 201-209.

Muñoz MES, De Giovanni R, Siqueira MF, Sutton T, Brewer P, Pereira RS, Canhos DAL \& Canhos VP (2011) openModeller: a generic approach to species' potential distribution modelling. GeoInformatica 15: 111-135.

Murray-Smith C, Brummitt NA, Oliveira-Filho AT, Bachman S, Moat J, Lughadha EMN \& Lucas
EJ (2009) Plant diversity hotspots in the atlantic coastal forests of Brazil. Conservation Biology 23: 151-163.

Nelson BW, Ferreira CA, Silva MFD \& Kawasaki ML (1990) Endemism centres, refugia and botanical collection density in Brazilian Amazonia. Nature 345: 714-716.

Pearson RG (2007) Species distribution modeling for conservation educators and practitioners. Synthesis, American Museum of Natural History 1: $1-50$.

Pearson RG, Raxworthy CJ, Nakamura M \& Peterson AT (2007) Predicting species' distributions from small numbers of occurrence records: a test case using cryptic geckos in Madagascar. Journal of Biogeography 34: 102-117.

Peterson AT, Papes M \& Soberón J (2008) Rethinking receiver operating characteristic analysis applications in ecological niche modeling. Ecological modelling 213: 63-72.

Phillips SJ, Anderson RP \& Schapire RE (2006) Maximum entropy modeling of species geographic distributions. Ecological Modelling 190: 231-259.

Procópio LC \& Secco RDS (2008) The importance of botanical identification in forest inventories: the example of "tauari"- Couratari spp. and Cariniana spp., Lecythidaceae - in two timber areas of the State of Pará. Acta Amazonica 38: 31-44.

Quinet A (2005) Sinopse taxonômica da família Lauraceae no estado do Rio de Janeiro, Brasil. Acta Botanica Brasilica 19: 563-572.

Quinet A (2006) Lauraceae na Reserva Biológica de Poço das Antas, Silva Jardim, Rio de Janeiro, Brasil. Rodriguésia 57: 543-568.

Rabinowitz D, Cairns S \& Dillon T (1986) Seven forms of rarity and their frequency in the flora of the British Isles. In: Soulé ME (ed.) Conservation biology. The science of scarcity and diversity. Sinauer Associates, Sunderland. Pp. 82-204.

RLBF (2016) Lista vermelha da flora do Brasil. Disponível em <http://cncflora.jbrj.gov.br>. Acesso em março 2016.

Rupprecht F, Oldeland J \& Finckh M (2011) Modelling potential distribution of the threatened tree species Juniperus oxycedrus: how to evaluate the predictions of different modelling approaches? Journal of Vegetation Science 22: 647-659.

Schulman L, Toivonen T \& Ruokolainen K (2007) Analysing botanical collecting effort in Amazonia and correcting for it in species range estimation. Journal of Biogeography 34: 1388-1399.

Serrato A, Ibarra-Manríquez G \& Oyama K (2004) Biogeography and conservation of the genus Ficus (Moraceae) in Mexico. Journal of Biogeography 31: 475-485. 
Siqueira MF, Durigan G, Marco Júnior P \& Peterson AT (2009) Something from nothing: using landscape similarity and ecological niche modeling to find rare plant species. Journal for Nature Conservation 17: 25-32.

Swets K (1988) Measuring the accuracy of diagnostic systems. Science 240: 1285-1293. van der Werff H (1987) A revision of Mezilaurus (Lauraceae). Annals of the Missouri Botanical Garden 74: 153-182.

Yamaguchi KKDL, Alcântara JM \& Veiga Junior VFD (2012) Antioxidant and anticholinesterasic effects of 20 species of the family Lauraceae. Acta Amazonica 42: 541-546. 